\title{
sit \\ Para una genealogía de la anormalidad: la teoría de la degeneración de Morel
}

Sandra GAPoni

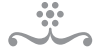

RESUMEN

Analizamos aquí la teoría de la degeneración de Morel tal como fue definida en su Tratado de degeneración de la especie humana, publicado en 1857 . Ese texto surge como una tentativa de dar respuesta a las dificultades con las que la naciente psiquiatría debía enfrentarse. A partir de ese momento, comenzó a construirse ese suelo epistemológico que posibilitó la creación y la posterior consolidación de una psiquiatría ampliada que, aún hoy, parece obcecada en clasificar y patologizar los más variados comportamientos. La teoría de la degeneración crea un marco explicativo amplio de la enfermedad mental, en el interior del cual desaparecen las barreras taxativas entre alienación y degeneraciones menores, definidas como desvíos físicos o morales del tipo primitivo. A partir de ese momento, la psiquiatría sale de los muros del asilo para dedicarse a la gestión completa del espacio social.
\end{abstract}

Palabras-clave • Degeneración. Alienación mental. Historia de la psiquiatría. Foucault.

Psiquiatría ampliada.

\section{INTRODUCGIÓN}

El Manual Diagnóstico y Estadístico de Trastornos Mentales (DSMIV), editado en cuarta edición en el año de 1992, en sus 886 páginas, clasifica 297 diagnósticos psiquiátricos. La nueva edición del DSMIV, en preparación, multiplicará el número de diagnósticos psiquiátricos de acuerdo a síntomas clínicos bastante ambiguos, supuestas etiologías y nuevas terapéuticas, que pueden ser o no farmacológicas. Una inmensa literatura hoy está dedicada a estudiar los limites y las dificultades de esa proliferación de diagnósticos que han posibilitado que casi todos nuestros sufrimientos puedan ser redefinidos en términos médicos (cf. Horwitz, 2002; Moynihan \& Cassels, 2006; Conrad, 2007). Las fronteras entre lo normal y lo patológico son cada vez más móviles e inestables; la medicalización de conductas consideradas como socialmente indeseables, hoy clasificadas como anormales, se ha banalizado y extendido a todos los dominios de nuestra existencia. El DSMIV incluye diversos tipos de demencia, desde la esquizofrenia y el trastorno bipolar hasta trastornos de sueño, la depresión, la fobia social o hiperactivi- 
dad, pasando por diagnósticos extremamente ambiguos, tales como, la "desorden matemática" - "mathematical disorder (315.1)" - o la "desorden de lectura" - "reading disorder (315.00)" (APA, 1992).

Para intentar comprender que es lo que permite agrupar en un mismo espacio clasificatorio diagnósticos tan diversos y heterogéneos como la esquizofrenia, la depresión y los más virados desordenes de comportamiento, será necesario observar las condiciones históricas que permitieron la construcción de ese suelo epistemológico, en el interior de cual pudo surgir una psiquiatría ampliada referida tanto a los delirios como a las conductas cotidianas.

Se dirá que esas clasificaciones encuentran su legitimidad en los recientes avances de la neurociencia, en los nuevos aparatos de diagnóstico por imagen como los pet scan, también se atribuirá el refuerzo y multiplicación de esos diagnósticos a la industria farmacéutica, que está dedicando cada vez más atención al inmenso negocio representado por los psicofármacos. Sin embargo, mi objetivo no es analizar aquí el surgimiento y las controversias ocurridas en el ámbito de las neurociencias o de la psicofarmacología, sino comprender ese momento histórico en el que se produce una nueva configuración epistemológica, que permite que la psiquiatría se transforme en un espacio de saber y de intervención que es, al mismo tiempo, intra y extra asilar. Doron (2009) formula algunas preguntas referidas a los trastornos de personalidad que son relevantes para este estudio:

¿Cómo se construyó esa idea según la cuál existe cierto tipo de locura - o mejor
de trastorno - que ya no se caracteriza por ideas delirantes, dificultades de imagi-
nación, sino por actos y comportamientos considerados socialmente aberrantes,
aunque la razón pueda ser considerada sana? ¿Cómo surge esa figura de la perso-
nalidad anormal que reagrupa todos los pequeños desvíos de conducta de un in-
dividuo desde su infancia, quien se va a ocupar de la reparación, de la recupera-
ción para prevenir un peligro social? Cuestiones foucaultianas, sin duda (Doron,
2009, p. 4).

A partir de la publicación del Tratado de degeneración de la especie humana de Morel, en 1857, las imágenes de irracionalidad, peligro, delirio y alucinación, asociadas en las mentes de los alienistas a las figuras del loco y de la locura, comenzaron a vincularse con nuevos sujetos que, poco a poco, irán integrándose al saber psiquiátrico y a una pesada red de intervenciones correctivas: los anormales.

La medicalización de los anormales, a la que Michel Foucault (1999) dedica el curso del College de France del año 1974-1975, no puede ser comprendida fuera del marco de la "teoría de la degeneración". Ian Hacking (2000) se refiere a esa teoría como siendo 
Para una genealogía de la ANormalidad: LA teoría de la degeneración de Morel

un verdadero “programa de investigación”, que concentró los esfuerzos de médicos, higienistas, juristas y psiquiatras en torno a una misma preocupación: definir y establecer tanto los alcances como los limites de la idea de anormalidad y "degeneración".

Esa obsesión por la degeneración posibilitó la aparición y definición de nuevas y variadas enfermedades mentales: desde las manías de persecución, pasando por los exhibicionismos y el alcoholismo, hasta la ninfomanía o la cleptomanía entre muchos otros trastornos. En los Anales de higiene y medicina legal y en los Anales médico-psicológicos, vemos multiplicarse los estudios sobre nuevas patologías de degeneración. En el primer caso, verificamos que los estudios sobre la degeneración se extienden hasta el año 1919; en el segundo, encontramos un discurso algo mas critico ya en el año 1896, aunque los estudios sobre degeneración continúan sucediéndose hasta el año 1926. Estos textos relatan tipos diversos de "locura de degeneración", amplían el cuadro nosológico con la definición de nuevas enfermedades mentales vinculadas entre sí a partir de la teoría de la degeneración.

En los Anales médico-psicológicos del día 16 de junio de 1896, aparece una crítica a la teoría de la degeneración bastante ambigua dirigida a rescatar la importancia del trabajo de Morel (1857) y Magnan (1893), como teoría antropológica y patogénica, pero no como método nosológico. El autor afirma:

Considero esta teoría aplicable a la patología general y no a las que poseen caracteres especiales. Los mismos estigmas físicos y psíquicos pertenecen a todas las locuras hereditarias en general y no a una sola. Son características comunes a la gran familia de los degenerados y no a una especie o variedad particular. Mi opinión sobre la teoría de la degeneración es que engloba en un gran cuadro las formas más variadas de locura. Representa, para mí, como un inmenso océano, sin limites y sin fondo en el cual vienen a desaparecer todas las variedades, todas las especies, aún las mejor establecidas, de enfermedad mental (Arnauld, 1896, p. 437$)$.

Para intentar definir ese océano inmenso que abarca todas las enfermedades mentales y que permite situar en un mismo espacio teórico y clasificatorio, entre otras patologías, las alucinaciones, los delirios, el exhibicionismo y la persecución, será necesario analizar el texto que da inicio a esas reflexiones teóricas. Me refiero al Tratado de degeneración de la especie humana de Benedict August Morel, publicado en el año 1857 . 


\section{El gongepto de degeneración de Morel}

La segunda mitad del siglo xix parece haber estado marcada por el aumento creciente de alienados internos en asilos y por un sentimiento de fracaso en relación a las promesas terapéuticas de la psiquiatría clásica (cf. Pinell, 2006, p. 312). El "tratamiento moral" de Pinel y Esquirol (cf. Ackerknecht, 1982) parecía insuficiente para resolver los problemas de esa población, designada como alienada, que no dejaba de multiplicarse.

Por otra parte, existían grandes dificultades para llegar a un consenso sobre el mejor modo de establecer parámetros de clasificación para las patologías mentales, que pudieran llegar a ser tan confiables como las clasificaciones de patologías orgánicas, construidas a partir de la localización de lesiones en el cuerpo. La clasificación propuesta por Pinel partía de la idea de unicidad de la enfermedad mental, afirmando que la misma podía manifestarse de modos diferentes, dando lugar a cuatro clases fundamentales de alienación: la manía, la melancolía, el idiotismo y la demencia. Siguiendo esa misma concepción de enfermedad mental, Esquirol amplió el cuadro nosológico de alineaciones, diferenciando: manía, lipemanía, monomanía, idiotismo y demencia. Aunque esas eran las clasificaciones más utilizadas, en realidad se trataba de diferentes modos de aparición de una misma patología que era la alienación mental. En consecuencia, todas esas manifestaciones podían ser tratadas del mismo modo. Ante una misma enfermedad se imponía una misma terapéutica: el tratamiento moral (cf. Lantéri-Laura, 1999, p. 103).

La teoría de la degeneración de Morel surge como una tentativa de dar respuesta a esas dificultades con las que la naciente psiquiatría debía enfrentarse. Fundamentalmente, el tratado de Morel se propone crear una clasificación creíble y consistente de las patologías mentales, que no esté basada solamente en la diferenciación de síntomas (generalmente muy similares unos a otros) o de manifestaciones corporales exteriores (los estigmas que pueden llevar a clasificaciones erradas). Morel pretendía sustituir una clasificación sintomática por una clasificación etiológica de las enfermedades mentales. Pues, como afirma una y otra vez en su tratado, solo cuando estén claramente determinadas las causas se podrá crear una red clasificatoria de nosologías y una terapéutica o profiláctica apropiada para cada patología. Y es a partir de esa red causal explicativa que Morel crea el principio general de toda su obra: "los seres degenerados forman grupos y familias con elementos distintivos relacionados invariablemente a las causas que los transformaron en eso que son: un desvío mórbido del tipo normal de la humanidad" (Morel, 1857, p. 75).

La preocupación por la degeneración de los organismos, por el desvío del tipo originario de la especie, está presente en el discurso de naturalistas como Buffon o Lamarck. Esa concepción de la degeneración de las especies, propia de la historia na- 
tural, fue transformada a fin del siglo xviıı, cuando Cabanis (1802) comenzó a asociarla con una nueva mirada médica. A partir de ese momento, la degeneración pasará a ser aplicada a modificaciones indeseadas en los tejidos y órganos, articulándose, de ese modo, el discurso de la anátomo-patología con las teorías clásicas de los naturalistas.

En la historia natural del siglo xvıı, la idea de degeneración remitía casi exclusivamente a la problemática de las razas y se vinculaba fundamentalmente con la cuestión climática. Esa idea se mantendrá durante gran parte del siglo XIX, como lo muestran los trabajos de geografía médica de Boudin (1857). Hasta allí la problemática de la degeneración o de la variabilidad de las especies y las razas parecía encontrar en el medio externo, particularmente en las consecuencias derivadas de un clima extremo, una explicación casi exclusiva de las causas de la degeneración tanto de los animales como de las razas humanas. La idea de "pesimismo climático", directamente asociada a las empresas colonialistas, se mantendrá inalterada por décadas reproduciendo, una y otra vez, la idea difundida por Boudin de una causa privilegiada de las patologías mentales y orgánicas, difundidas en las regiones tropicales colonizadas por los europeos blancos. Esa idea permanecerá más o menos inalterada en el discurso de muchos médicos pasteurianos enviados para las colonias de ultramar aún a inicios del siglo xx.

En todos esos casos, a excepción de Cabanis, la causa de la degeneración debía ser buscada en el medio externo, particularmente en los efectos nocivos que el clima tórrido producía en los cuerpos de los individuos, ya sea por la creación de razas degeneradas, o porque producía estados patológicos (mentales o físicos) en los europeos blancos, que no lograban aclimatarse a un clima diferente de aquel donde habían nacido. Las consecuencias malsanas del clima tórrido estaban vinculadas con una serie de elementos asociados a las altas temperaturas: el mefitismo del aire, los efluvios palúdicos, las intoxicaciones, la fatiga crónica. Las degeneraciones se manifestaban en el cuerpo por marcas exteriores como el color de piel, la altura, el tipo de cabello y por marcas comportamentales como la pereza, la indolencia y las alienaciones mentales que se agravaban con el tiempo de permanencia en los trópicos. En la medida en que se reconoce una causa privilegiada para las degeneraciones, la terapéutica podía limitarse simplemente a la restitución al clima de origen, a la disminución del tiempo de permanencia de soldados y colonos en las colonias de clima tórrido, el control de las bebidas alcohólicas y de la alimentación. En los casos en los cuales la degeneración de la raza había llegado a extremos irrecuperables, solo restaba intentar rescatar a los descendientes, si aún no habían sido seriamente afectados. La degeneración se transmitía de generación en generación mientras las condiciones climáticas se mantuvieran adversas, cambiadas esas condiciones, el organismo recuperaba su estado natural. 


\section{La GLASIFICACIÓN ETIOLÓGIGA DE LAS ENFERMEDADES MENTALES}

Ese es el contexto en el que surge el Tratado de degeneración de Morel donde se defiende una posición contraria, tanto a las ideas de los naturalistas que afirmaban una causa climática única de los procesos de degeneración, como a la psiquiatría de Pinel y Esquirol, quienes diferenciaban los tipos de locura por referencia a la sintomatología y no a la etiología (cf. Ackerknecht, 1982).

Para Morel, que postula la existencia de una pluralidad de causas de la degeneración, era preciso clasificar las patologías en familias y grupos nosológicos de acuerdo con la causa predominante. Entonces se podría proponer una terapéutica adecuada, en el caso de tratarse de degeneraciones reversibles, y se podría anticipar, por vía de acciones de profilaxis y de higiene, la ocurrencia de padecimientos irreversibles.

De igual modo, y aproximándose en este punto a Cabanis (que no es mencionado en el tratado), Morel no comparte con los naturalistas la idea de que los signos exteriores, que establecen diferencias entre las razas, puedan ser considerados como indicadores de degeneración de la especie. El color de piel, el tipo de cabello, o la altura no indican más que variaciones del tipo normal que no tienen por que ser consideradas patológicas. Las variaciones entre razas humanas son diferentes de las variaciones degenerativas, mientras las razas pueden procrearse, esto es, son fértiles, los individuos o familias que degeneraron están condenados a la esterilidad después de cuatro generaciones.

Así, aunque el clima pueda producir alteraciones en los organismos, esas alteraciones no son necesariamente patológicas, esto es, no deben ser necesariamente consideradas como formas degeneradas. El clima y los fenómenos asociados, ya sea el aire mefítico o las emanaciones palúdicas, no son más que una de las causas delimitadas por Morel. Si recordamos la distinción de la higiene clásica establecida por Hallé entre "circunfusa" e "ingesta" (cf. Jorland, 2006; Cartron, 2000, p. 13), veremos que Morel no se limita a los elementos que clásicamente forman parte de la "higiene circunfusa" - la temperatura, el suelo pantanoso, el aire mefítico, los espacios mal ventilados -, sino que dará cierta preeminencia a los elementos clásicamente referidos a la "higiene ingesta" - a las intoxicaciones por plomo, a los alimentos ingeridos, a los efectos nocivos de las drogas como el hachís o el opio, como lo indican los informes dejados por los misionarios que habitaron en China.

Pero, entre todas esas causas intoxicantes, existe una que aparece como una fuerte amenaza y que provoca las formas de degeneración más graves y radicales, es la ingestión desmedida de alcohol cuyas consecuencias son analizadas en el tratado de Morel, que se refiere tanto a las alteraciones que ocurren en el cuerpo de los individuos alco- 
Para una genealogía de la ANormalidad: LA teoría de la degeneración de Morel

hólicos, como a las alteraciones que surgirán en las generaciones sucesivas de familias afectadas por ese mal, para detenerse, por fin, en las sociedades y pueblos condenados a la degeneración por abuso de alcohol. Destaca, en este último caso, las consecuencias irreversibles de lo que considera un pueblo sin futuro, por estar condenado a la más absoluta degeneración: los habitantes de Suecia.

Como vemos con el ejemplo del pueblo sueco, no es en función de las características físicas de las diferentes razas que puede designarse grados de degeneración. Por el contrario, para Morel, "entre el estado intelectual de un bosquimano salvaje y el del europeo más avanzado de su civilización, hay menos deferencia que la que existe entre el estado intelectual de ese mismo europeo y el de un ser degenerado" (Morel, 1857, p. 46). El bosquimano es susceptible de modificación y sus descendientes podrán inclusive llegar a transformarse en el "tipo perfecto", por el contrario, un ser degenerado solo podrá tener alguna modificación relativa pero las influencias hereditarias fatales tendrán efectos nocivos sobre los descendientes. Las generaciones futuras se mantendrán toda su vida como lo que en realidad son: "un espécimen de degeneración de la especie humana, un ejemplo de desvío malsano del tipo normal de la humanidad" (Morel, 1857, p. 47).

Digamos algunas pocas palabras sobre ese "tipo normal de la humanidad". En realidad, ese ser efectivamente inexistente no es otra cosa que un postulado metafísico. Se trata del sujeto primitivo, creado por Dios, para perpetuar la especie humana de manera siempre idéntica. Morel es heredero de ese pensamiento religioso que recupera los mitos del génesis y del pecado original, y son esas ideas las que le permiten defender la tesis de un tipo primitivo, que se constituye como obra maestra y resumen de la creación. Todo desvío de esa obra maestra representará inevitablemente una degradación no solo física sino también moral de la humanidad.

Ese espíritu religioso se encuentra lado a lado con una mirada que pretende ser científica y organicista, una mirada médica impregnada de los desarrollos de la anátomo-patología de su tiempo. Y si esta complementariedad es posible se debe a que, según afirma Patrice Pinell (2006, p. 310), aún los presupuestos que le permitieron construir una aproximación organicista a la enfermedad mental eran de orden metafísica. El mismo punto de vista religioso que lo llevó a pensar a la locura como la caída después del pecado original, lo condujo, por referencia a la idea de consustancialidad entre alma y cuerpo, a tratar del mismo modo a todas las causas de la degeneración sean ellas físicas o morales, sea que afecten al cuerpo o al alma. 


\section{Causas y tipos de degeneración}

Para Morel, los procesos de degeneración sólo pueden ser el resultado de influencias mórbidas, de orden física o moral, que siempre responden a ciertas características generales y a ciertas características específicas. La causa general, que aparece necesariamente en todos los casos, sea como elemento predisponente, sea como efecto de causas exteriores, es la transmisión hereditaria. La idea de herencia de Morel se diferencia, como veremos más tarde, tanto de las teorías clásicas existentes hasta el momento, que suponían una repetición en los descendientes de las mismas conductas y de los mismos delirios que aparecían en sus ancestrales, como de las teorías de los alienistas que imaginaban una herencia difusa donde la existencia de cualquier hecho patológico podía llevar a los descendientes al delirio o a la locura, como lo ejemplifica Foucault (2003) con la idea de "cuerpo ampliado". La herencia es una de las causas, sin duda la más importante de degeneración, aunque en la enumeración de los procesos causales ella aparezca mencionada en último lugar.

Morel distingue las siguientes causas: (1) Degeneraciones por intoxicación; él incluye en este grupo la mayor parte de las causas climáticas destacadas por los naturalistas, como el suelo pantanoso, las epidemias, los miasmas palúdicos, el aire viciado, siendo que entre todos esos factores se destaca el abuso de alcohol. (2) Degeneraciones resultantes del medio social, como las producidas por actividades industriales, la miseria y las profesiones insalubres. Morel se refiere aquí específicamente al conjunto de circunstancias complejas que tiende a modificar desfavorablemente a las clases pobres, claramente se refiere a la asociación, que ha marcado todo el siglo xix, entre clases pobres y clases peligrosas. (3) Degeneraciones que resultan de una afección mórbida anterior o de un temperamento malsano. El conjunto de degeneraciones producidas por esta causa da lugar a todo ese grupo de enfermos que habita en los hospitales psiquiátricos: los que tienen delirios epilépticos, las histéricas, los hipocondríacos, aquellos que poseen ideas delirantes, los maníacos, los melancólicos, los que sufren de parálisis general, los que padecen idiotismo e imbecilidad. En todos esos casos, es preciso hablar de una predisposición hereditaria para que estas enfermedades puedan aparecer.

Esta confusa enumeración de redes causales que se superponen continúa con las (4) degeneraciones derivadas de la inmoralidad. Tomando como punto de partida la indisoluble relación entre lo físico y lo moral, esta red causal se refiere específicamente a las degeneraciones físicas que derivan de males morales. La vinculación estrecha entre alma y cuerpo impide que se pueda estudiar de modo abstracto e independiente las influencias morales e intelectuales, sin especificar las consecuencias y transformaciones que esos hechos puedan tener en los organismos afectados. Para Morel, nece- 
Para una genealogía de la Anormalidad: LA teoría de la degeneración de Morel

sariamente existirán ciertas marcas en los cuerpos, ciertos estigmas que son, por así decir, los trazos biológicos de la inmoralidad. En quinto lugar, Morel analiza las (5) degeneraciones que provienen de enfermedades congénitas o adquiridas en la infancia. Estas causas provocan padecimientos que aparecen en la infancia como consecuencia de un desarrollo deficitario del sistema nervioso que se manifiesta a edad temprana, como en el caso de los retardos mentales y del idiotismo congénito. Esto puede ocurrir por un defecto del cerebro, constitutivo o provocado por ingestión de sustancias tóxicas del seno de la madre o como consecuencia de enfermedades como la tuberculosis o las convulsiones. En este grupo, Morel sitúa también a los ciegos y sordomudos, porque la privación de un sentido físico puede tener consecuencias fatales para el desarrollo moral.

Por fin, se referirá a las (6) degeneraciones que están en relación con las influencias hereditarias. Destaca que esta es la causa más general y universal, pues está presente, de algún modo, en todas las formas de degeneración. En relación a las enfermedades mentales, Morel sostiene que, después de varias generaciones de alienados, la degeneración se transforma en incurable. Por esa razón, el tratamiento moral dedicado a la alienación mental ha llevado a tantos y tantos fracasos. En palabras de Morel:

las condiciones de degeneración, en las cuales se encuentran los herederos de ciertas disposiciones orgánicas viciosas, no sólo se revelan por características exteriores fáciles de descubrir, como la baja estatura, la conformación defectuosa de la cabeza, el predominio de un temperamento malsano, las deformidades, las anomalías orgánicas y la imposibilidad de reproducirse, sino aún más profundamente en las más extrañas aberraciones en el ejercicio de las facultades intelectuales y de los sentimientos morales (Morel, 1857, p. 62).

Esas causas pueden afectar a los individuos, familias y poblaciones de manera más o menos profunda. Las degeneraciones pueden ser pasajeras o crónicas, dependiendo de si las causas que las provocaron son efímeras o permanentes, pueden ser congénitas o adquiridas, completas o incompletas, susceptibles de cura o incurables. El cretinismo es considerado como la degeneración por excelencia, los cretinos forman una familia que se caracteriza por tener las mismas aptitudes intelectuales, las mismas tendencias instintivas y las mismas características físicas exteriores.

Esa intrincada red causal, confusa y plagada de reiteraciones, fue, sin embargo, la estructura explicativa básica que permitió que la teoría de la degeneración se mantuviera con algunas variaciones por más de 50 años. En esos años, muchas de las causas identificadas por Morel, así como muchas categorías o familias de degenerados fueron substituidas y abandonadas. Sin embargo, muchas de esas explicaciones permanecie- 
ron y se profundizaron con los trabajos posteriores de Magnan (1893). Los teóricos de la degeneración que sucedieron a Morel aceptaron el postulado de Cabanis de la indisoluble relación entre lo físico y lo moral, se mantuvieron fieles a la teoría de la herencia y buscaron en la anátomo-patología cerebral explicaciones cada vez mas precisas para la intuición de Morel sobre el papel central que ejerce el sistema nervioso y el cerebro en la degeneración hereditaria. Por fin, las referencias a los estigmas y al cachet typique, que podemos traducir como la marca típica de los degenerados, se mantuvieron casi inalteradas durante todo el siglo xIx y parte del siglo xx.

\section{EL GEREBRO: ENTRE EL ALMA Y EL GUERPO}

No es posible analizar las causas de degeneración sin observar el papel desempeñado por el cerebro y por el sistema nervioso en esa articulación compleja que, para Morel, existe entre la dimensión física y la dimensión moral o intelectual del hombre (cf. Lantéri-Laura, 1999).

El cerebro ocupa en esa estructura explicativa un papel articulador. Es, al mismo tiempo, un elemento metafísico que permite vincular cuerpo y alma, y un elemento orgánico cuyas lesiones pueden ser observadas en los cadáveres de los alienados, siguiendo rigurosos métodos de anatomía y patología comparada. Ocupa, digámoslo resumidamente, el mismo lugar que la glándula pineal ocupaba en Descartes, una bisagra entre dos mundos, uno extenso y uno pensante. Solo que aquí, la articulación se dará entre dos órdenes del ser: el intelectual o moral y el físico u orgánico. No se trata ni en Descartes ni en Morel de un alma que, con el auxilio de la glándula pineal o del cerebro, se transformaría en una guía para el cuerpo. Al contrario, para Morel, el sistema nervioso, y las lesiones cerebrales, son aquello que permite tejer una unidad indisoluble y absoluta entre nuestros pensamientos o decisiones morales y nuestro organismo, esto es, entre nuestra alma y nuestro cuerpo.

Por esa razón, dará un gran destaque a lo que denomina de causas mixtas (cf. Morel, 1857 , p. 55), causas que son al mismo tiempo físicas y morales. Esto significa que es posible identificar tanto causas físicas que tienen como efecto afecciones morales, como inversamente, factores intelectuales y morales que afectan determinados órganos y tejidos y que tienen impacto directo en la creación de diferentes clases de degeneración, aquí se trata de "degeneraciones físicas que provienen de un mal moral" (Morel, 1857, p. 54). Afirma que un gran número de observaciones rigurosas lo han llevado a concluir que "no solo es difícil, sino imposible estudiar separadamente las causas exclusivamente morales y exclusivamente físicas” (Morel, 1857, p. 56). 
Para una genealogía de la ANormalidad: LA teoría de la degeneración de Morel

Uno de los ejemplos privilegiados es el de los efectos nocivos que producen la miseria y las degradantes condiciones de vida de la sociedad industrial, que alteran profundamente los temperamentos y los organismos. "Si observamos la influencia desmoralizadora que ejerce la miseria, la falta de instrucción, el abuso de alcohol, el exceso de enfermedades, la falta de alimento, tendremos una idea de las circunstancias complejas que tienden a modificar de una manera desfavorable los temperamentos de la clase pobre" (Morel, 1857, p. 51). Sin embargo, Morel no acepta las teorías de los economistas entonces difundidas que bregaban por resolver los problemas derivados de la miseria por la modificación de las condiciones materiales de existencia. Su perspectiva de estudio, como insiste en afirmar, es la del médico y no la del economista. Esa concepción ingenua supone un saber médico capaz de dar respuesta a los problemas sociales, económicos, morales, intelectuales y humanos, por referencia a explicaciones fundadas en lesiones orgánicas o en prácticas médicas regeneradoras, basadas en intervenciones higiénicas y moralizantes.

Los hechos sociales, los efectos de miasmas palúdicos o la ingestión de elementos tóxicos importan en la medida que dejan sus marcas en los organismos, produciendo las más variadas degeneraciones. Si en relación a las alienaciones mentales los estudios clínicos de sus pacientes ya lo habían llevado a concluir que los delirios epilépticos, las manías, la histeria, las ideas delirantes, la melancolía, y las parálisis generales coinciden, en cada caso, con un mismo déficit funcional del organismo, ahora podrá ampliar esa misma reflexión para los temperamentos y los comportamientos moralmente indeseados. Estos temperamentos extremos pueden aparecer como resultado de un déficit funcional orgánico o como causa (indicada en tercer lugar) de un tipo de degeneración donde existen disfunciones orgánicas derivadas de un temperamento malsano.

Recordemos que cuando Morel analiza las degeneraciones derivadas de la inmoralidad, enumerada como la cuarta causa, no duda en afirmar que "el cerebro es el órgano del alma" (Morel, 1857, p. 56). Es por eso que las degeneraciones, sean ellas causadas por hechos morales o físicos, siempre tendrán como referencia alguna alteración en el sistema nervioso o algún desorden cerebral. Aún en el interior de un discurso fuertemente religioso, Morel encuentra un límite orgánico para las posibilidades espirituales:

Toda fuerza, aún la espiritual, está limitada por este órgano (el cerebro), nada puede ser hecho, nada puede ser producido, fuera de las posibilidades delimitadas por este instrumento. El alma puede tener conciencia de los limites impuestos por el cerebro pero no podrá sobrepasarlos (Morel, 1857, p. 57). 
El cerebro predispone a los individuos para determinadas actividades, pensamientos o actos. Aunque no podamos realizarlos plenamente por dificultades del medio, nunca podremos superar los limites impuestos. En ciertos casos, esos limites se evidencian en la infancia, cuando al nacer los niños poseen un cerebro incapaz de cumplir su función, sea por estar atrofiado en su estructura íntima o porque la estructura ósea del cráneo es demasiado pequeña para posibilitar su desenvolvimiento posterior (cf. Morel, 1857, p. 58). Esto limitará no solo el desenvolvimiento intelectual sino también el desenvolvimiento moral y físico. En otras oportunidades, las lesiones cerebrales no resultan evidentes en la infancia y aparecen en la vida adulta como efecto de predisposiciones hereditarias o de factores externos.

Así, para Morel, como para Cabanis, aunque son retomadas algunas ideas hipocráticas, existe una relación directa entre los temperamentos y las lesiones cerebrales. Para la tradición médica heredera de Hipócrates, un temperamento solo puede considerarse enfermo cuando es excesivo, como es el caso del temperamento melancólico que puede transformarse en una patología. En el caso de Morel, lo que determina si un temperamento debe ser considerado como una patología o degeneración es la persistencia de la causa. Si esta última es efímera, producirá patologías pasajeras y curables, si es permanente, la patología será crónica e incurable.

Un temperamento es un conjunto de propiedades fisiológicas y de aptitudes morales vinculadas directamente con el funcionamiento de los órganos. Existe una relación íntima y necesaria entre ciertos tipos de degeneración y ciertas lesiones del sistema nervioso que están, a su vez, vinculadas a cierto tipo de temperamento patológico. Estos temperamentos se repiten en determinadas familias, creando degeneraciones que se suceden en los descendientes, de modo que "tal temperamento, tal actitud intelectual y moral, tal cualidad o defecto físico, son característicos de ciertas familias y aún de ciertas razas" (Morel, ${ }_{1857}$, p. 65). Lo que demuestra al mismo tiempo el carácter anatómico, cerebral, de los temperamentos y su carácter hereditario.

\section{ESTIGMA Y HERENGIA DISIMILAR}

Para Pinell, "el punto fuerte de la teoría de Morel expuesta en el Tratado de 1857, en todo caso el punto de mayor interés para sus contemporáneos, es el tipo de vínculo que establece entre desorden cerebral y transmisión hereditaria" (2006, p. 311). A diferencia de Esquirol y Pinell, interesados en la herencia de patologías semejantes, la preocupación de Morel estará en la "herencia disimilar" (Cartron, 2000, p. 13). Para explicar la herencia como causa de degeneración, Morel utiliza tres nociones fundamentales: la predisposición, las causas predisponentes y las causas determinantes. El pro- 
Para una genealogía de la ANormalidad: LA teoría de la degeneración de Morel

ceso se inicia, como explica Pinell, con las causas predisponentes, esto es, con hechos morales o físicos que pueden provocar padecimientos mentales que, aún siendo poco graves, necesariamente serán transmitidos a los descendientes. Ellos tendrán mayor predisposición para que se desencadene una enfermedad nerviosa ante la presencia de una causa determinante, que puede ser interna o externa, física, social o moral. En presencia de esa causa, necesariamente se producirá una enfermedad mental severa que será, a su vez, transmitida a los descendientes como una predisposición hereditaria. Ese proceso de degeneración se manifestará de maneras diferentes, aunque con gravedad creciente, en las sucesivas generaciones.

La concepción de Morel no es idéntica a las teorías clásicas de herencia por semejanza, porque lo que se repite no es la misma enfermedad sino ciclos de predisposición y manifestaciones patológicas más o menos previsibles pero siempre cambiantes.

El ejemplo que reaparece como tipo paradigmático, como caso ejemplar de la degeneración en la obra de Morel, es el alcoholismo. La primera parte del tratado de Morel está dedicada al análisis de los efectos que el alcohol produce en los individuos, familias y pueblos. Dirá que el alcoholismo crónico es una enfermedad que permite observar el desarrollo de diversas lesiones en el sistema nervioso, desde las que se manifiestan como un simple temblor, hasta las que producen parálisis general. El alcoholismo puede producir el grado más extremo de degradación al que el hombre puede llegar, en la medida que produce la abolición completa de todos los sentimientos morales. Diferentes estudios anatómicos y patológicos demostraron la influencia nefasta de la intoxicación por alcohol en el sistema nervioso. Así, las lesiones cerebrales evidenciadas por la abertura de cadáveres de alcohólicos ponen en evidencia la gravedad de esa situación y el fin prematuro al que están condenados estos enfermos (cf. Morel, 1857 , p. 80).

Tanto con relación al alcohol como al opio se puede concluir que: "Las causas más activas de degeneración de la especie humana son aquellas que atacan directamente el cerebro, produciendo estados especiales y llevando periódicamente a quien hace uso de estos agentes tóxicos a la condición de locura momentánea” (Morel, 1857 , p. 334).

La transmisión hereditaria de padecimientos del sistema nervioso y de lesiones cerebrales provoca alteraciones en el encéfalo de los descendientes, produciendo enfermedades que se suceden con tal regularidad que parecen cumplir el ciclo fatal de grados diferentes de degeneración (cf. Morel, 1857, p. 335). Para Morel, el descendiente de un alcohólico no será necesariamente un alcohólico, ni se repetirán las mismas lesiones cerebrales. Puede ocurrir, por el contrario, como muestran las observaciones clínicas de Morel, que la descendencia sea abstemia y que las lesiones en el sistema nervioso sean de un tipo diferente. "Los desvíos del tipo normal de la humani- 
dad, que aparecen en las generaciones sucesivas, se revelan por signos interiores y exteriores mucho más alarmantes, con un debilitamiento aún mayor de las facultades mentales y morales" (Morel, 1857, p. 324). Se inicia así un ciclo de patologías que sobrevendrán unas a las otras. Uno de los casos analizados muestra que la primera generación de alcohólicos, caracterizada por excesos, depravación y inmoralidad fue sucedida por una segunda generación que padecía de ebriedad hereditaria, accesos maníacos y parálisis general; la tercera generación, aunque se caracterizaba por la sobriedad, poseía ideas persecutorias y homicidas; la cuarta llevaba las marcas del idiotismo, la estupidez y la falta de inteligencia, estando condenada por su esterilidad a ser él último representante de esa familia (cf. Morel, 1857, p. 125).

Esas historias patológicas, propias de las familias de degenerados, llevan a Morel a concluir que, para comprender los procesos de alienación y degeneración, no es posible limitar la observación a las lesiones en los cadáveres. Entiende que será necesario ampliar los estudios anátomo-patológicos con consideraciones sobre la predisposición hereditaria y las transformaciones cíclicas que afectan a las diferentes generaciones. Para poner en evidencia la llamada herencia disimilar se deberá estudiar la historia de vida, o la historia patológica, de cada una de las generaciones que pertenecen a las diferentes familias de degenerados. Solo así será posible identificar clases y categorías de degeneración, para poder así establecer una clasificación confiable, etiológica y no sintomática, de las patologías mentales.

Pero, al mismo tiempo que las explicaciones etiológicas permiten establecer clases de degeneración de acuerdo a lesiones internas y a predisposiciones hereditarias, ellas permiten crear categorías de individuos cuyas semejanzas externas pueden servir como indicadores de diferentes tipos de degeneración. Morel llama a esas características de cachet tipique o stigmata. Son marcas o signos de degeneración que caracterizan a las diferentes familias y grupos de degenerados. Los teóricos de la degeneración que sucedieron a Morel, como Magnan o Broca (1867), reforzaron aún más la importancia de los estigmas. En el Tratado de 1857, aunque la referencia a indicadores externos es casi una constante, se afirma que es preciso no identificar esas marcas con las verdaderas causas. Morel afirma:

Yo insisto sobre ese punto, porque la observación rigurosa de los hechos me ha llevado a concluir que el desvío del tipo normal no consiste exclusivamente en las diferencias exteriores que son en apariencia tan importantes (p. 37).

Los elementos distintivos no descansan solamente en las diferencias exteriores, sino en los desvíos interiores que provienen del grado menor o mayor de perfección del sistema nervioso y de los aparatos sensitivos (p. 71). 
Para una genealogía de la Anormalidad: LA teoría de la degeneración de Morel

Muchas veces, los signos exteriores pueden crear confusiones para el método clasificatorio. En ciertos casos, las diferencias físicas pueden llevar a agrupar como perteneciendo a clases diferentes individuos que poseen las mismas lesiones cerebrales y, en consecuencia, el mismo tipo de afección. En otros casos, la semejanza de estigmas físicos puede llevar a agrupar en una misma categoría sujetos que padecen enfermedades diferentes. Resumidamente, "existen caracteres generales que pertenecen a diferentes categorías de seres degenerados, pero también existen caracteres exteriores especiales que distinguen una variedad de degeneración de otra" (Morel, 1857, p. 71 ).

En los casos extremos, las marcas exteriores resultan más evidentes como ocurre con el cretinismo. Esa forma extrema de degeneración posee características propias que se manifiestan en la forma de la cabeza, la altura que no puede sobrepasar un cierto límite, un tipo particular de rostro, pero también se manifiesta en las mismas aptitudes intelectuales y en las mismas tendencias instintivas que son características de esa familia de degenerados.

\section{Degeneración y ALIENAGión MENTAL}

Resta establecer de cual modo Morel articulaba el territorio de las alineaciones mentales y de los delirios con ese espacio, extendido y de fronteras difusas, que es el de las familias afectadas por algún tipo de degeneración. Para comprender esa relación es necesario recordar una afirmación que se repite una y otra vez en el Tratado: la alienación mental no es otra cosa que un estado avanzado de degeneración (cf. Morel, 1857, p. $77,344,347$ ). Morel se pregunta:

Que son los asilos de alienados sino la concentración de las principales degeneraciones de la especie humana? A partir del momento que un enfermo es colocado en ese lugar con un certificado de maníaco, de lipemaniaco, de epiléptico, de demente con parálisis, de idiota o de imbécil, encontramos en la mayor parte de los casos, sino en todos, el producto de una de las causas de degeneración que aquí nos ocupan (Morel, 1857, p. 77).

Allí es posible apreciar los efectos devastadores que el exceso de alcohol, la miseria, las privaciones, las profesiones insalubres y las condiciones morales degradadas dejan en los cuerpos de los individuos y en su descendencia. Según Morel, solo es posible comprender la existencia de esta "masa enorme de seres incurables confina- 
dos en los asilos" (Morel, 1857, p. 78), si se entiende que ellos son el último eslabón, el más degradado, de la cadena de degeneraciones que ha marcado a una familia.

La alienación es el punto final, muchas veces irreversible, de la sucesión de degeneraciones heredadas por familias afectadas de las más diversas patologías. Para cada uno de los habitantes de los asilos, es posible rastrear las causas predisponentes (por ejemplo, un padre alcohólico) y las causas determinantes (miseria, degradación moral), observar lesiones cerebrales (por necropsias) y relacionarlas con dificultades intelectuales o morales, clasificar a los enfermos de acuerdo al conjunto de causas que provocaron las diferentes patologías, identificar marcas o estigmas físicos semejantes para cada clase, pero aún así, difícilmente será posible hablar de cura o recuperación. Los asilos albergan sujetos condenados.

Nunca, desde el origen de esa institución médica, fueron tantos los esfuerzos destinados a recuperar a los desdichados alienados. ¿Cómo explicar ese estado de cosas, cuando el número de curas obtenidas está lejos de responder a las legítimas esperanzas de los sabios y al progreso del sistema hospitalario? (Morel, 1857, p. 344).

Para Morel, los alienistas se han negado a aceptar que la enfermedad mental no puede ser curada, en la generalidad de los casos, por representar el punto final al que se llega después de generaciones afectadas por degeneraciones físicas y mentales. Para él no se trata de permanecer en la trampa del asilo y la locura, se trata de encontrar signos anunciadores de una locura por venir, de anticipar los delirios en los desvíos de comportamiento de aquellos que hoy presentan pequeñas anormalidades, que sí pueden ser tratadas.

De ese modo, las estrategias terapéuticas propuestas por Morel deberán diferenciarse de las defendidas por los alienistas cuyas intervenciones se limitaban al momento de la crisis maníaca, delirante o melancólica que provocó el internamiento psiquiátrico. En palabras de Morel:

Desde que intenté, ya hace algunos años, salir del círculo estrecho creado por la alienación mental, desde que procuré una vía nueva en los estudios de las causas que producen la degeneración de la especie, incluida allí la alienación, no han dejado de estar estos esfuerzos sometidos a críticas y objeciones (Morel, 1857 , p. 357 ).

Salir del estrecho círculo de la alienación significaba inscribir a la crisis de demencia en un cuadro mayor que tiene su inicio, no solo en el momento del nacimiento, 
Para una genealogía de la ANormalidad: LA teoría de la degeneración de Morel

sino también en la genealogía de las familias afectadas por las más variadas anormalidades. Se inaugura así la psiquiatrización de las anomalías y los desvíos de comportamiento. Aquí, el tratado de Morel adquiere una asombrosa actualidad. Esa nueva psiquiatría propone la ampliación de las categorías nosológicas, que deberían pasar a formar parte de los asilos psiquiátricos y, al mismo tiempo, una psiquiatrización preventiva (llamada higiene moral) de las más variadas conductas cotidianas.

Morel distingue dos tipos de alienados confinados en los hospicios: aquellos que padecen de degeneración congénita, esto es, los idiotas, imbéciles, y los que tienen demencia con parálisis, y aquellos que son el resultado de una degeneración consecutiva o adquirida, esto es, los que padecen de manía, melancolía, idiotismo, lipemanía o demencia. Aunque los alienistas aceptaban el encerramiento psiquiátrico de los primeros, simplemente para protegerlos del abandono, no identifican estos casos con alienación mental. Morel entiende que esa distinción es innecesaria y artificial. Aunque los individuos que sufren de degeneración congénita no padezcan de delirios y no escuchen voces, representan, igual que los alienados, formas extremas de degeneración. Los retardados o idiotas deben ser clasificados como enfermos mentales junto con aquellos que sufren de manía o lipemanía, en consecuencia, el internamiento asilar de estos enfermos debe considerarse la mejor terapéutica.

Morel incluye también otras patologías en el conjunto de enfermedades mentales que deben llevar al internamiento psiquiátrico:

En la medida que la palabra degeneración posee una acepción amplia, extendiéndose a todos los que por una u otra causa se alejan más o menos del tipo normal de la humanidad, de igual modo quiero aplicar a un mayor número de variedades de enfermedad los beneficios de esas instituciones hospitalares. Yo no encuentro ningún inconveniente para que los sordo-mudos y ciegos de nacimiento, cuyas enfermedades congénitas están asociadas a causas degeneradoras de la especie, y cuyo estado intelectual, físico y moral presenta anomalías especiales (asociadas a esa deficiencia), sean admitidos en los mismos establecimientos que los alienados. Allí esos desdichados se transformarán en miembros útiles para la sociedad (Morel, 1857, p. 695).

A todas esas figuras les estaba destinada la terapéutica aplicada en los hospicios, llamada de profilaxis. La profilaxis se refiere a los casos extremos de degeneración, aquellos enfermos que deben ser secuestrados en asilos. Aunque la eficacia de esta institución se mostró extremamente limitada como espacio de cura, tiene una utilidad terapéutica para el entorno social del enfermo, permitiendo la realización de una profilaxis defensiva (cf. Morel, 1857, p. 690). El secuestro de individuos peligrosos o 
nocivos para los otros, que a diferencia de los delincuentes padecen una enfermedad, permite evitar daños a la familia y a la sociedad. Pero, los hospicios permiten también la realización de una profilaxis preventiva. "Pueden modificar las condiciones intelectuales, físicas y morales de aquellos que, por motivos diversos, fueron separados del resto de los hombres. Antes de reenviarlos a su medio social, la institución debe armarlos, por así decir, contra ellos mismos con la finalidad de atenuar las reincidencias" (Morel, 1857, p. 691).

Además de la profilaxis hospitalaria, Morel identifica otras estrategias terapéuticas, cada una de las cuales se destina a una variedad o tipo de degeneración. Estas estrategias son el tratamiento del estado agudo y el tratamiento moral.

El tratamiento del estado agudo no es otra cosa que la intervención médica destinada a resolver enfermedades físicas derivadas de causas sociales o morales. La intoxicación alcohólica, el abuso de narcóticos, los alojamientos insalubres, el aire mefítico modifican, como dijimos, el temperamento y se asocian a enfermedades agudas que precisan ser médicamente tratadas. Aún cuando los individuos no presenten signos de alienación mental, las enfermedades que padecen pueden afectar a las generaciones futuras produciendo degeneraciones en sus descendientes si no son debidamente asistidos.

Entre las tres estrategias terapéuticas definidas por Morel, la que parece ser más relevante es el tratamiento moral, y es justamente aquí que aparecen las mayores diferencias con las intervenciones de los alienistas restringidas al espacio asilar. Aunque utiliza el mismo concepto empleado por la psiquiatría clásica de Pinel y Esquirol, aquí se trata de intervenciones absolutamente diferentes. Morel afirma: "la palabra tratamiento moral, que empleamos en nuestros asilos para definir la acción que el médico ejerce sobre una fracción de degenerados, me parece una designación feliz" (Morel, 1857, p. 685). Se trata de la aplicación de una serie de reglas de higiene moral destinadas no solo a los alienados sino a las poblaciones en general, es lo que denomina moralización de las masas.

Entiende por tratamiento moral la aplicación y propagación de los deberes impuestos por una ley moral que es divina, fija e inmutable. Afirma que "la propagación de esa ley, su práctica, su aplicación a los individuos de acuerdo a su edad y grado de inteligencia no es una función exclusivamente reservada para algunos hombres" (Morel, 1857, p. 686). No deberían ser solamente los alienistas, los moralistas, los magistrados, sino también los padres de familia y cada uno de los miembros de la comunidad los que deberían difundir esa ley, sin embargo, para que eso pueda realmente ocurrir sería necesario que cada uno de ellos tuviera un profundo conocimiento, no solamente "del corazón humano, sino también un claro entendimiento de todas las anomalías que, bajo la influencia de causas degenerantes, se producen en las condiciones orgánicas e intelectuales del hombre" (Morel, 1857, p. 687). 
Para una genealogía de la ANormalidad: LA teoría de la degeneración de Morel

Aunque la ley moral acompaña a la humanidad desde su creación, el conocimiento metódico de la misma sólo podrá ser hecho a partir de una perspectiva médica. Es a partir de la mirada del anatomista y del patólogo, conjuntamente con las observaciones de los alienistas, que se podrá precisar en que sentido el organismo del hombre y las aptitudes fisiológicas de las generaciones futuras pueden modificarse por la aplicación de higiene moral. El tratamiento moral implica una ampliación absoluta y casi indefinida de la ingerencia de la psiquiatría. Ya no se trata, como en el tratamiento del estado agudo, de la cura de enfermedades derivadas de intoxicaciones, no se trata como en la profilaxis preventiva de moralizar a los alienados que se encuentran en el asilo, se trata de una profilaxis preventiva universal referida a todo y cualquier sujeto que pueda representar un desvío, por menor que este sea, del tipo primitivo de la humanidad.

\section{ConcLusión: HAGIA UNA PSIQUIATRÍA AMPLIADA}

La teoría de la degeneración inaugura un nuevo modo de observar y de analizar todo ese conjunto de fenómenos físicos y morales considerados como desvíos en relación al tipo primitivo, entre ellos las enfermedades mentales. Esa teoría tiene como objetivo prioritario redefinir las clasificaciones nosológicas de las patologías mentales, sin restringirlas al ámbito exclusivo de las alineaciones o los delirios. Considera que las clasificaciones de Pinel y Esquirol son insuficientes para dar cuenta de la amplitud del fenómeno de las patologías morales y mentales. Sin embargo, aún distanciándose de la psiquiatría clásica:

la teoría de la degeneración ha salvado a los alienistas que estaban buscando un fundamento organicista capaz de asegurarles una legitimidad científica y social para ejercer su trabajo. El proyecto del asilo había fracasado. Las órdenes religiosas que se habían ocupado anteriormente de los hospitales cuestionaban la gestión de los médicos y la competencia teórica de los alienistas sobre la locura (Serpa, 2006, p. 129).

La teoría de la degeneración ofrece para los alienistas un fundamento organicista que se refiere a lesiones cerebrales y a predisposiciones hereditarias. Permite también extender los dominios de la psiquiatría más allá de las fronteras del asilo. La identificación de redes causales y la clasificación etiológica de las degeneraciones, incluidas las alienaciones mentales, abre la posibilidad de detectar e interrumpir la sucesión patológica actuando directamente sobre los procesos causales de las alineaciones mentales. Esto significa que Morel consiguió "transponer el abismo que separaba a los 
alienistas del resto de la medicina. La aplicación de la teoría de la degeneración a la comprensión de la locura permitió las intervenciones de los alienistas fuera de los muros de asilo, teniendo por misión principal la de higienizar el tejido social y moralizar las masas" (Serpa, 129).

La aplicación de la teoría de la degeneración a las enfermedades mentales fue la estrategia discursiva que permitió que las más variadas conductas y hechos pudieran ingresar al ámbito de la psiquiatría. Podemos decir que, a partir de ese momento, comenzó a construirse ese suelo epistemológico que posibilitó la creación y la posterior consolidación de una psiquiatría ampliada que aún hoy parece obcecada en clasificar y patologizar los más variados comportamientos.

Si la teoría de la degeneración pudo mantenerse como referencia privilegiada del discurso psiquiátrico hasta inicios del siglo xx (cf. Foucault, 1999), fue porque ella permitió definir los argumentos básicos que dieron soporte teórico a la psiquiatría ampliada. La teoría de la degeneración crea un marco explicativo extenso de la enfermedad mental en el interior del cual desaparecen las barreras taxativas entre la alienación y las degeneraciones menores (desvíos físicos o morales del tipo primitivo). Cada caso de degeneración, del más leve al más grave, es visto como resultado de causas físicas (lesiones cerebrales), intelectuales, sociales y morales interrelacionadas, siendo que la causa principal de las enfermedades mentales será la predisposición degenerativa (mórbida) vinculada a la herencia patológica. En ese marco teórico el suceso de la psiquiatría estará dado por la prevención y la anticipación de la locura y su espacio de intervención privilegiado estará fuera de los muros del asilo, donde habitan los casos irreversibles.

Aunque muchas de esas premisas fueron abandonadas, aún hoy persiste la idea recurrente de que nuestros comportamientos y juicios morales, el modo como nos vinculamos con los otros y con nosotros mismos, está indisolublemente vinculado a lesiones o deficiencias que pueden ser localizadas en nuestro organismo, particularmente en nuestro cerebro, confiriéndose así una clara primacía al saber médico-psiquiátrico en la gestión de los asuntos humanos.@

Sandra Gaponi Professora Associada do Departamento de Saúde Pública, Universidade Federal de Santa Catarina. Pesquisadora do Conselho Nacional de Pesquisa Científica, CNPq, Brasil. sandracaponi@newsite.com.br 
Para una genealogía de la Anormalidad: LA teoría de la degeneración de Morel

\section{ABSTRACT}

Here we analyze Morel's theory of degeneration as it was presented in his Treatise of the degeneration of human species, published in 1857 . This text originated as an attempt to answer difficulties that had to be faced by the nascent psychiatry. Following it, there began to be constructed an epistemological ground that made possible the creation and the later consolidation of an extended psychiatry, which still today seems to be obsessed about classifying the most varied behaviors as pathologies. The theory of degeneration creates a very wide explanatory frame for mental illness, in which the clear distinction between alienation and minor degenerations disappears and both are understood as physical or moral detours of an original type. From this time on, psychiatry transcends the walls of the asylum, to devote itself to the complete management of the social space.

KEYwORDs $\bullet$ Degeneration. Mental alienation. History of the psychiatry. Foucault. Extended psychiatry.

\section{REFERENGIAS BIBLIOGRÁFIGAS}

Ackerkneght, E. Historia de la psiquiatría. Buenos Aires: Editora Universitaria de Buenos Aires, 1982.

Andrieu, B. (Org.). Dictionaire de corps dans les sciences humaines et sociales. Paris: CNRS, 2006.

Arnauld, D. Theorie des dégénérescences. Annales Médico-Psicologiques, 4, p. 435-8, 1896.

Apa. American Psyquiatric Association. Diagnostic and statistical manual Iv. 1992. Disponible en: <http:/

/www.psych.org/MainMenu/Research/DSMIV>. Acceso en: 17 abr. 2009.

Broca, P. Sur la prétendu dégénérescence de la population française. Paris: Martinet, 1867.

Boudin, J. Traité de géographie et de statistique médicale. Paris: Baillière, 1857.

Cabanis, P. J. Rapports du physique et du moral de l homme. Paris: Bailliére,1802.

Caponi, S. \& Lecointre, M. R. (Org.). Estudos de filosofia e história das ciências biomédicas. São Paulo:

Discurso, 2006.

Gartron, L. L'idée de dégénérescence au XIx $x^{e}$ siècle. Paris: EHESS, 2000.

Conrad, P. The medicalization of society. Baltimore: The Johns Hopkins University Press, 2007.

Doron, C. O. Archéologie des troubles de la personnalité. Seminaires du Centre Georges Canguilhem, 2009.

Disponible en: <http://centrecanguilhem.net/>. Acceso en: 15 mayo 2009.

Foucault, M. Les anormaux. Paris: Seuil, 1999.

. Le pouvoir psiquiatrique. Paris: Seuil, 2003.

Grmek, M. (Org.). Histoire de la pensée médicale en occident. Paris: Seuil, 1999. v. 3.

Hacking, I. Múltipla personalidade. Rio de Janeiro: José Olympio, 2000.

Honwtтz, A. Creating mental illness. Chicago: The University of Chicago Press, 2002.

JorLand, G. A higiene pública em França e Inglaterra: um estudo comparativo. In: CAPoni, S. \& Lecointre, M. R. (Org.). Estudos de filosofia e história das ciências biomédicas. São Paulo: Discurso, 2006. p. 255-76.

Lantéri-Laura, G. Le psychisme et le cerveau. In: Grmek, M. (Org.). Histoire de la pensée médicale en occident. Paris: Seuil, 1999. v. 3. p. 99-113.

Legourt, D. (Org.). Dictionaire de la pensée médicale. Paris: PUF, 2006.

Magnan, V. Leçons cliniques sur les maladies mentales. Paris: Baillére, 1893.

Morel, B. Traité des dégénérescence de l'éspèce humaine. Paris: Baillére, 1857.

Moynihan, R. \& Cassels, A. Medicamentos que nos enferman: la industria farmacéutica que nos convierte en pacientes. Barcelona: Contrapunto, 2006.

Pinell, P. Dégénérescence. In: Lecourt, D. (Org.). Dictionaire de la pensée médicale. Paris: PUF, 2006.

Serpa, O. D. Dégénérescence. In: Andrieu, B. (Org.). Dictionaire de corps dans les sciences humaines et sociales. Paris: CNRS, 2006. 